\title{
La metodología de cuidados en el abordaje al cansancio del cuidador del paciente incidente en programa de hemodiálisis
}

Trinidad López Sánchez* - Ma Luz Amoedo Cabrera* - Francisco Javier Toro Prieto** Marta Pérez Doctor* - Ma Carmen Chozas López*** -

*Enfermera - **Nefrólogo - ***Auxiliar de enfermería

U.G.C Nefrourológica. Hospitales Universitarios "Virgen del Rocío". Sevilla

\section{Sra. Directora:}

La entrada en programa de hemodiálisis (HD) puede afectar al bienestar y la satisfacción del paciente/cuidador por los cambios de la nueva situación de salud y el manejo del tratamiento que determinan los factores relacionados de los diagnósticos de enfermería ( $D d E$ ) "Cansancio en el desempeño de rol del cuidador" o "es vulnerable a ello" la clasificación NANDA define el primero como: "La persona percibe o demuestra dificultad para desempeñar el papel de cuidador de la familia". Estos diagnósticos, por su prevalencia observada en nuestra práctica diaria con estos pacientes, fueron incluidos en el Plan de Cuidados Estandarizado de los enfermeros de nuestra unidad hospitalaria con el objetivo general de mejorar el bienestar, la preparación y la satisfacción final del binomio paciente-cuidador, preparando el traslado al centro periférico de HD; nuestros objetivos específicos fueron:

- Identificar con metodología de cuidados la frecuencia de estos diagnósticos.

- Valorar la modificación de los objetivos propuestos con las intervenciones enfermeras puestas en marcha en nuestro plan de cuidados.

\begin{tabular}{|c|}
\hline Correspondencia: \\
Trinidad López Sánchez. \\
U.G.C Nefrourológica \\
Hospitales Universitarios "Virgen del Rocío" \\
Avda. Manuel Siurot s/n \\
41013. Sevilla \\
trilopez8@hotmail.com
\end{tabular}

\section{Material y método}

Se trata de un estudio descriptivo transversal retrospectivo de los pacientes que iniciaron un programa de HD durante el año 2007, atendidos por un total de 28 enfermeros de HD que trabajan con metodología de cuidados desde el año 2006 y con asignación primaria de cuidados o de enfermero referente según el proceso enfermero.

Se registró en tablas codificadas los datos recogidos mediante entrevistas al paciente y/o cuidador, y de las historias de enfermería archivadas. Se analizaron la edad, el sexo y la procedencia. Además de otos datos clínico y sociodemográficos se consideró:

a) Una valoración Inicial Integral (figura 1) en la que se tuvo en cuenta la comunicación del paciente, seleccionando a los que no tenían dificultad de comprensión y expresión y la capacidad del cuidador principal, valoración de la dificultad y/o incapacidad para ejercer el rol de cuidador.

b) El Plan de Cuidados (figura 2) en el que se activó el DdE: "Cansancio en el desempeño de rol del cuidador" con una selección focalizada de dos criterios de resultados:

1. Criteriol: Bienestar del cuidador principal con los indicadores satisfacción con la salud física y satisfacción con el apoyo profesional.

2. Criterio 2: Preparación del cuidador familiar domiciliario con los indicadores: voluntad de asumir el rol de cuidador familiar y conocimientos 


\begin{tabular}{|c|c|c|c|c|c|}
\hline \multicolumn{5}{|c|}{ 8. Patrón Rol/Relaciones: } & \multirow{2}{*}{ Deterior. comunicac. verbal } \\
\hline \multirow[t]{2}{*}{ Comunicación } & $\square$ Sin dificultad & $\square$ D. comprensión & $\square$ D. escritura & $\square$ D. lectura & \\
\hline & $\square$ D. expresión & $\square$ Otros idiomas & $\square$ Laringuectomiz & $\square$ Traqueotomía & Aislamiento social \\
\hline $\begin{array}{l}\text { Cuidador ppal. } \\
\text { Capacidad: }\end{array}$ & $\square$ Sin dificultad & $\square$ Dificultad & $\square I$ & ccapacidad & $\begin{array}{l}\text { Deterioro } \\
\text { Interacción social }\end{array}$ \\
\hline Observaciones: & & & & & $\begin{array}{l}\text { Interrupción procesos familia } \\
\text { Cuestionarios, índices, test } \\
\text { (sugeridos): } \\
\text { - C.A. Familiar, C. Zarit }\end{array}$ \\
\hline
\end{tabular}

Figura 1. Registro del patrón relaciones en la Valoración Inicial Integral de M. Gordon

\begin{tabular}{|c|c|c|c|}
\hline $\begin{array}{l}00062 \text { Riesgo de cansacio } \\
\text { del rol de cuidador r/c: } \\
\square \text { Duración de la necesidad de cuidados } \\
\text { y cuidados numerosos y complejos } \\
\square \text { Deterioro de la salud del cuidador, } \\
\text { falta de descanso y distracción } \\
\square \text { El cuidador no está preparado para } \\
\text { asumir el rol } \\
\text { Código A/R }\end{array}$ & $\begin{array}{l}\text { Escala Likert 1: } \\
\text { 1. No del todo satisfecho } \\
\text { 2. Algo satisfecho } \\
\text { 3. Moderadamente satis- } \\
\quad \text { fecho } \\
\text { 4. Muy satisfecho } \\
\text { 5. Completamente satis- } \\
\text { fecho }\end{array}$ & $\begin{array}{l}\text { Escala Likert 2: } \\
\text { 1. Inadecuado } \\
\text { 2. Ligeramente adecuado } \\
\text { 3. Moderadamente ade- } \\
\text { cuado } \\
\text { 4. Sustancialmente ade- } \\
\text { cuado } \\
\text { 5. Completamente ade- } \\
\text { cuado }\end{array}$ & $\begin{array}{l}\text { Escala Likert 3: } \\
\text { 1: } \\
\text { 2: } \\
\text { 3: } \\
\text { 4: } \\
\text { 5: }\end{array}$ \\
\hline Intervenciones de Enfermería (NIC) & \multicolumn{3}{|c|}{ Criterios de resultados (NOC) } \\
\hline \multirow{2}{*}{7040 Apoyo al cuidador principal } & & & \\
\hline & \multicolumn{3}{|c|}{2508 Bienestar del cuidador principal (1) } \\
\hline \multirow[t]{5}{*}{7110 Fomentar la implicación familiar } & \multicolumn{3}{|c|}{ Satisfacción de la salud física } \\
\hline & \multicolumn{3}{|c|}{ Satisfacción con el apoyo profesional } \\
\hline & \multicolumn{3}{|c|}{2202 Preparación del cuidador familiar domiciliario (2) } \\
\hline & \multicolumn{3}{|c|}{ Voluntad de asumir el rol del cuidador } \\
\hline & \multicolumn{3}{|c|}{ Cumplimiento de cuidados en el seguimiento } \\
\hline
\end{tabular}

Figura 2. Registro en el Plan de Cuidados Estandarizado (NANDA/NIC/NOC)

de los cuidados en el seguimiento. Todos estos indicadores son revisados periódicamente en las evoluciones de enfermería según escala liker de mejora ascendente de 1 a 5 .

c) El Informe de continuidad de cuidados (ICC) (figura 3) al alta con registro de la activación de los DeE "Afrontamiento familiar comprometido", "Afrontamiento familiar incapacitante" y "Síndrome de estrés por traslado", anotando las causas y manifestaciones del paciente y del cuidador al alta.

El análisis estadístico se llevó a cabo con el programa SPSS 15.0.

\section{Resultados}

Durante el año 2007 iniciaron HD 150 pacientes, de los cuales el $42,67 \%$ eran mujeres y el $57,33 \%$ hombres, con una edad media de 60,67+/-16,25. Del total de los pacientes el $61,54 \%$ procedían del área de prediálisis. Quedaron fuera de la muestra 12 pacientes por fallecimiento, tres por traslado a DP y uno por recuperación de función renal.

Con respecto a los objetivos propuestos:

1. El $4 \%$ de los pacientes, fueron valorados a través del cuidador por dificultad manifiesta de compren- 
8 PATRÓN ROL/RELACIONES: NOC:

$\square$ Aislamiento social r/c:

$\square$ Riesgo

$\square$ Cansancio en el desempeño del rol del cuidador r/c:

$\square$ Afrontamiento familiar comprometido/incapacitante $r / c$ :

Necesita cuidador: $\square$ SÍ $\square$ NO En contacto con SS.

Sociales $\square$ Sí $\square$ NO $\square$ Apoyo al cuidador principal

$\square$ Apoyo a la familia

$\square$ Derivación

$\square$ Asesoramiento

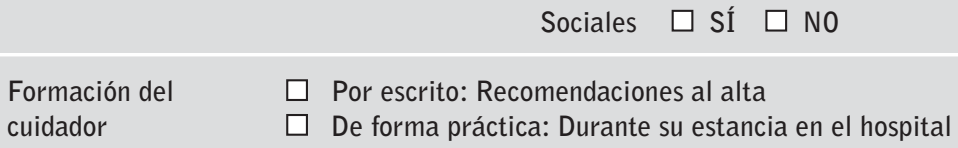

Cuidador Principal en el domicilio:

1 PATRÓN ADAPTACIÓN/TOLERA: CIA NOC:

\section{O AL ESTRÉS}

Riesgo

Manifestaciones del

paciente:
Síndrome de estrés al traslado r/c:

Deterioro de la adaptación R/C:

Nervioso
Aumentar el afrontamiento

$\square$ Aumentar los sistemas de apoyo

Figura 3. Informe de continuidad de cuidados

sión de los mismos. Un 24\% de los cuidadores tenían dificultad y/o incapacidad para ejercer su rol.

2. El diagnóstico de enfermería."Cansancio en el desempeño del rol de cuidador", se activó en el $48 \%$ los pacientes y descendió al alta en un $28 \%$ aunque se mantuvo el riesgo en el $61,2 \%$ de los casos resueltos (figura 4 ).

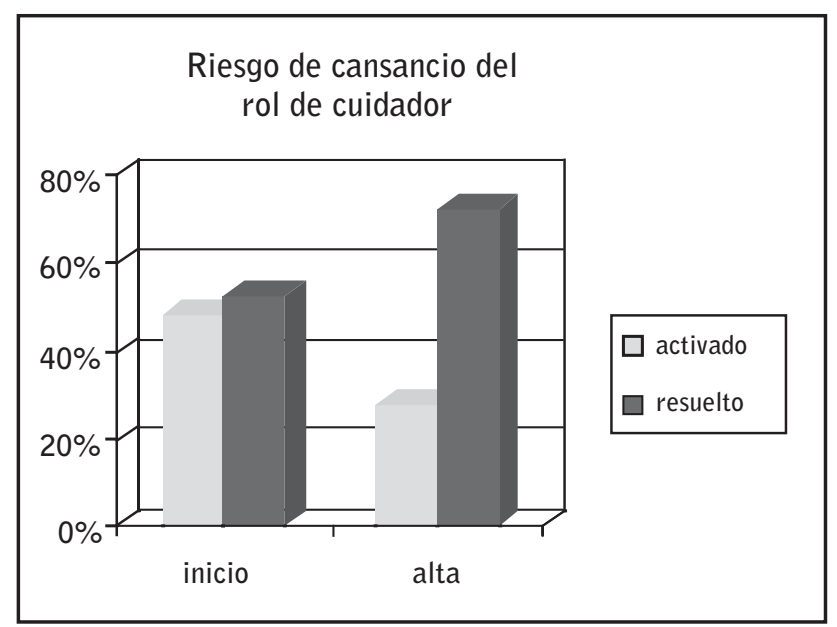

Figura 4. Evolución del diagnóstico
3. Respecto al criterio NOC "Bienestar del cuidador principal", en el indicador que contempla la salud del cuidador, con las intervenciones NIC puestas en marcha en el Plan de Cuidados, la satisfacción pasa de ser moderada (liker 3) a muy satisfecha (liker 4), pasando de un $33 \%$ a un $41,3 \%$, con la resolución del diagnostico en el $17,6 \%$. El alivio manifestado se debía fundamentalmente a la intervención sobre el temor/ansiedad inicial, con poca mejoría cuando existía afectación de la salud física del cuidador. Respecto al indicador "Apoyo profesional" la satisfacción del cuidador, mejora de moderada (liker 3), a sustancial o completa en (liker 4-5) pasando del $60 \%$ inicial al $87 \%$ al alta, debido principalmente al manejo del régimen terapéutico y el aumento del afrontamiento, aunque la intervención persiste activada al alta en un $65,33 \%$. En un $76 \%$ no se alcanzó la satisfacción completa por causas ajenas, como las demoras en los traslados, problemas con las ambulancias, etc. Aspectos que fueron registrados en un libro de incidencias de la unidad.

4. Respecto al criterio NOC "Preparación del cuidador familiar". En el indicador "Voluntad de asumir el rol" se mejora en la satisfacción de un nivel sus- 
tancial (liker 4), a satisfacción completa (liker 5), pasando del $80 \%$ al $96 \%$. Esta mejoría está muy relacionada con el indicador "Conocimiento de los cuidados en el seguimiento" que mejora de moderada (liker 3)) a sustancial (liker 4) pasando de $69,6 \%$ al $89 \%$. Esto se debe fundamentalmente a las intervenciones de enseñanza de dieta, medicación y proceso de la enfermedad. Pocas veces lo consideramos resuelto por los refuerzos necesarios en estos pacientes con altibajos en el estado de salud y frecuentes reajustes en el plan terapéutico.

5. Se activaron los DdE "Afrontamiento familiar comprometido" y "Afrontamiento familiar incapacitante" en un $27,8 \%$ de pacientes al alta se detectó un riesgo de cansancio del rol de cuidador, mientras que el 38\% manifestaban la necesidad de un cuidador. Fue preciso contactar con los servicios sociales en un $10,3 \%$ por presentar un síndrome sociosanitario con precisión de continuidad de cuidados con atención primaria (figura 5).

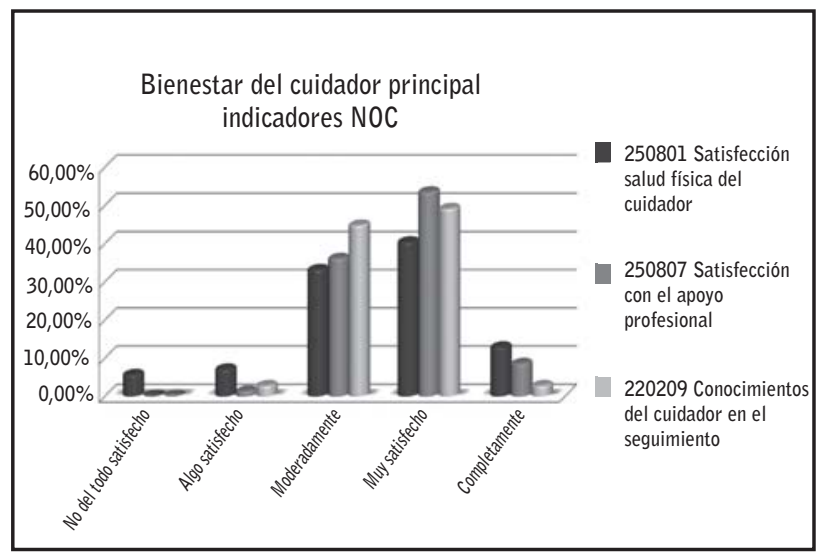

Figura 5. Evolución del criterio

6. Un $98,6 \%$ recibieron recomendaciones al alta por escrito y el $98 \%$ de forma práctica durante un promedio de 10-15 sesiones tras las que se estaban contentos y animados por los beneficios del traslado. Sin embargo, en un 38,6\% re registró el diagnóstico de "Riesgo de estrés por traslado" con manifestaciones de desconfianza, preocupación y falta de seguridad al alta que nos hizo dudar de la eficacia de nuestras intervenciones (figura 6 y 7); no obstante, expresaban que el motivo de este estrés era la inseguridad e incertidumbre de ser atendido en el centro periférico con las mismas intervenciones que habíamos realizado en nuestro plan de cuidados.

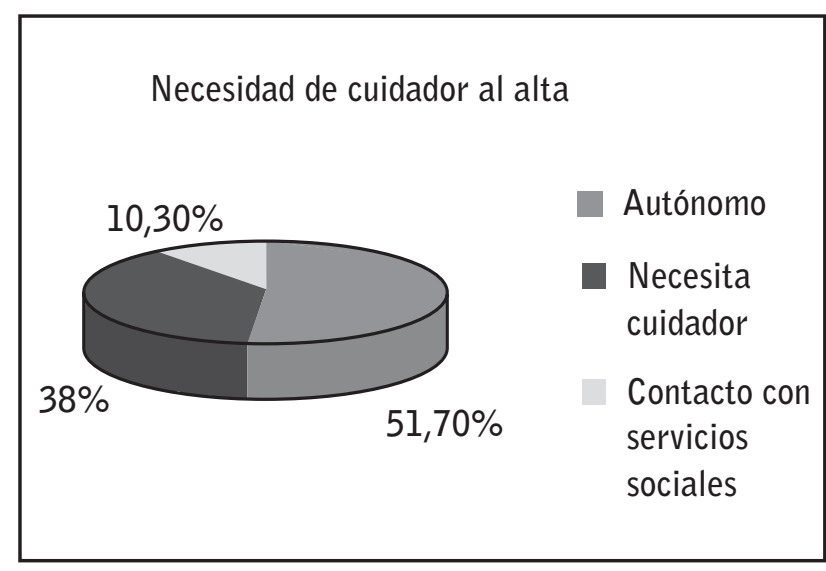

Figura 6. Evolución del criterio

Síndrome de estrés por traslado al alta

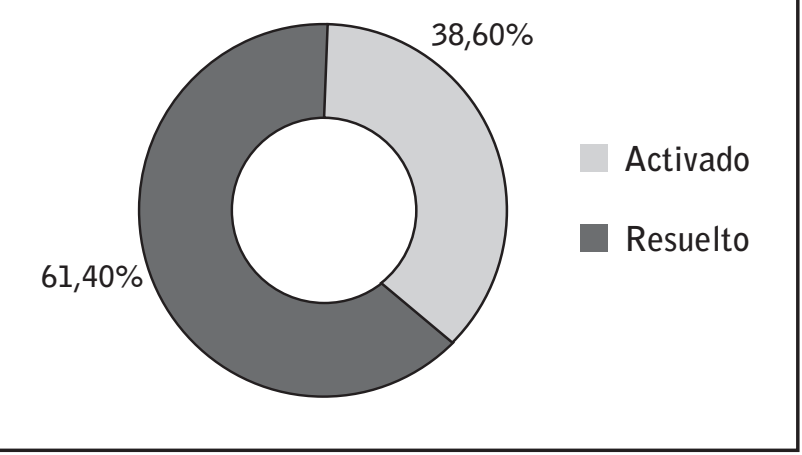

Figura 7. Evolución del diagnóstico

\section{Conclusiones}

La inclusión del cansancio del cuidador en el abordaje de los problemas de salud del paciente incidente en HD pone de manifiesto su alta vulnerabilidad para el desempeño del rol de cuidador principal. Es necesario, por tanto, establecer un nuevo marco relacional entre los profesionales y los cuidadores principales, reconociendo su influencia determinante en la salud y pronóstico de los pacientes que inician programa de HD. 
La gestión sistemática y con metodología (NANDA/NIC/ NOC) de los cuidados ofrecidos mejora el cansancio, preparación y satisfacción final del paciente/cuidador, garantiza la continuidad y responde a sus expectativas según los Principios de Calidad de nuestro centro hospitalario y unidad de gestión.

\section{Bibliografía}

1. NANDA. Diagnósticos enfermeros: Definiciones y clasificación 2007-2008. Elsevier; 2008.

2. McCloskey J, Bulechek G.Clasificación de Intervenciones de Enfermería (NIC). Elsevier; 2005.

3. Morread S, Johnson M, Maas M. Clasificación de Resultados de Enfermería (NOC). Elsevier; 2005.
4. Sociedad Española de geriatría y gerontología. Cuidar a los que cuidan. Centro de psicología aplicada Universidad Autónoma de Madrid. (Recuperado mayo 2005). http://www.uam.es/centros/psicologia/ paginas/cuidadores/informacion_programas.html.

5. Cuevas Cancino JJ. Adaptación del cuidador principal a la agonía de uno de los padres. Rev. Desarrollo Científico Enfermero México 2003; 11(9):262-267.

6. Pérez PJ. Cuidadores informales en un área de salud rural: perfil, calidad de vida y necesidades. Biblioteca Las casas, 2005; 1. Disponible en http://www.index-f. com/lascasas/documentos/lc0015.php.

7. Ríos OA, Rueda LG, Valverde OA, Mohamed HF. Cansancio en el desempeño del rol de cuidador. Metas de Enfermería. 2001; 4(7):30-36. 